\title{
Uber seismische Wellen in anelastischen Material
}

\author{
H. Menzel $(*)$
}

Received on September 15 th, 1977.

Rinssunto - Viene studiata la propagazione di onde sismiche in un mezzo inclastico. La relazione tra il tensore tensione e quello deformazione d̀ della forma:

$$
\gamma \sigma_{i j}+\beta_{i i}=\left(i_{0}^{\prime} \varepsilon_{k k} \hat{o}_{i j}+-\mu^{\prime} t_{i j}\right)+\lambda \varepsilon_{k k} \delta_{i j}+z \mu \varepsilon_{i j}
$$

Sono calcolate la velocità di propagazione c l'estinzione delle onde di compressione e di quelle di taglio.

$\mathrm{Si}$ suppone che l'eccitazione delle onde sia puramente armonica c che agisca lungo una linea della superficie.

SUMmARY - The propagation of seismic waves in inelastic media is investigated. The relation between stress and strain tensor is of the form

$$
\gamma \sigma_{i j}+\dot{\beta}_{i j}=\left(\lambda^{\prime} \varepsilon_{k k} \delta_{i j}+=\mu^{\prime} t_{i j}\right)+\lambda \varepsilon_{k k} \delta_{i j}+z \mu \varepsilon_{i j}
$$

Tehe velocity of propagation and the extinction both of compressional and shear-waves are calculated. The cxitation of the waves is assumed to be purely harmonic and acting at a line of the surface.

Die einfache Beziehung zwischen Spannungs - und Deformationstensor, welche durch das Hooksche Gesetz angegeben wird, gilt nicht mehr in einen anelastischen Körper.

Vielmehr spielen dann noch die zeitlichen Ableitungen dieser Tensoren eme Rolle. Sehr allgemein kann man

$$
F\left(\sigma_{i j}, \dot{\sigma}_{i j}, \ddot{\sigma}_{i j}, \ldots, \varepsilon_{i j}, \dot{\varepsilon}_{i j}, \ddot{\varepsilon}_{i j} \ldots\right)=0
$$

(*) Institut für Cicophysik. Universitit Hamburg. 
setzen. [1] Die spezielle Form dieses Gesetzes hängt von den physikalischen Vorgängen $a b$, die bei der Untersuchung anelastischen Verhaltens in Betracht gezogen werden.

Nimmt man an, dass $\sigma_{i j}$ und $\varepsilon_{i j}$ so klein sind, dass Produkte dieser Grössen vernachlässigt werden können, und nimmt man ferner an, dass $\ddot{\sigma}_{i j}, \ddot{\varepsilon_{i j}}$ und höhere zeitliche Ableitungen nicht berücksichtigt werden müssen, so kann man bei Isotropie aus [1] die Beziehung

$$
\gamma \sigma_{i j}+\dot{\sigma}_{i j}=\lambda^{\prime} \varepsilon_{k k} \delta_{i j}+z \mu^{\prime} \varepsilon_{i j}+\lambda \dot{\varepsilon}_{k k} \delta_{i j}+\rho \mu \dot{\varepsilon_{i j}}
$$

entwickeln. Aus ihr folgt:

$$
\left.\sigma_{i j}=\lambda \varepsilon_{k k} \delta_{i j}+z \mu \varepsilon_{i j}+\int_{-\infty}^{t}\left[\left(\lambda^{\prime}-j \lambda\right) \varepsilon_{k k} \delta_{i j}+\rho \mu^{\prime}-j \mu\right) \varepsilon_{i j}\right] e^{-\gamma(1-z)} d z
$$

(s. a. [2], [3], [4], [5]).

Gleichung (2a) zeigt, dass der Zusammenhang zwischen $\sigma_{i j}$ und $\varepsilon_{i}$, bei adiabatischen und isothermen Vorgängen in dass Hooksche Gesetz übergeth. Dabei ist dann

$$
\lambda=\lambda_{A d l .,} \quad \mu=\mu_{k d .}, \frac{\lambda^{\prime}}{\gamma}=\lambda_{i s .}, \frac{\mu^{\prime}}{\gamma}=\mu_{i s .}
$$

Aus [2b] folgt

$$
\sigma_{k k}=3 k \varepsilon_{k k}+3 \int_{-\infty}^{\ell}\left(k^{\prime}-\gamma k\right) \varepsilon_{k k} e^{-\gamma(t-z)} d z
$$

mit

$$
k=\lambda+\frac{z}{3} u \text { und } k^{\prime}=\lambda^{\prime}+\frac{z}{5} \mu
$$

Das Integral auf der rechten Seite von [3], bzw. [2b] stellt die Abweichungen von ideal-elastischem Verhalten dar. Es ist eine Beobachtungstatsache, dass Abweichungen von idealer Elastizität sich hauptsächlich bei Scherungen, aber sehr wenig bei Kompressionen bemerkbar machen. Nehmen wir der Einfachheithalber, und um die durch Beobachtungen zu bestimmenden Konstanten zu vermindern, an, dass bei reinen 
Kompressionen gar keine Abweichungen von ideal-elastischem Verhalten auftritt. Dann muss

gelten. Hieraus folgt

$$
k^{\prime}-\gamma k=\rho
$$

$$
\lambda^{\prime}-\gamma \lambda=-\frac{z}{3}\left(\mu^{\prime}-\gamma \mu\right)
$$

Es wird

$$
-\frac{z}{3}\left(\mu^{\prime}-\gamma \mu\right)=\beta
$$

gesetzt. Das liefert:

$$
\sigma_{i j}=\lambda z_{k k} \delta_{i j}+z \mu \varepsilon_{i j}+\beta \int_{-\infty}^{l}\left(\varepsilon_{k k} \delta_{i j}-3 \varepsilon_{i j}\right) e^{\cdots \cdot(1-\cdots)} d z
$$

Es soll übrigens bemerkt werden, dass $\beta$ wie auch $\gamma$ positiv sein muss.

Dann folgt aus [5]

$$
\frac{\beta}{\rho}=\frac{z}{3} \gamma\left(b^{i_{k t}}{ }-b_{i s}\right)
$$

Die Scherwellengeschwindigkeit ist im adiabatischen Fall also grösser als im isothermen. Für den Unterschied der Kompressionswellengeschwindigkeiten erhält man:

$$
a_{k d}^{2}-a_{i s}^{i}-2-\frac{\rho}{\rho \gamma} \text {. }
$$

Er ist also grösser als jener der Scherwellengeschwindigkeiten. Es soll nun folgendes Problem behandelt werden:

An der ebenen Grenzfläche eines homogenen und isotropen, aber anelastischen Halbraumes werde eine Störspannung erzeugt. Sie wirke gleichmässig auf einer beiderseits unendlich ausgedehnten geraden Linie und beginne zu einem festen Zeitpunkt. Ihr Zeitverlauf sei eine harmonische Schwingung konstanter Frequenz. 
Das Problem ist zweidimensional zu behandeln. Die $x_{1}$-Achse liege in der Oberfläche, die $x$-Achse stehe auf dieser Fläche senkrecht.

Der Nullpunkt des Koordinatensystemes liege in der Geraden, in der die Störspannung wirkt.

Es gelten die Gleichungen

$$
\rho=\frac{\partial i u_{i}}{\partial t^{z}}=\frac{\partial \sigma_{i j}}{\rho x_{i}}(\mathrm{i}=\mu z)
$$

und für $x,=0$

$$
\sigma_{i z}=-N_{i}\left(t, x_{z}\right), \quad x_{z}=0
$$

Dabei sei $N_{1}\left(t, x_{2}\right)$ die Störspannung und die Normale auf der Fläche $x_{i}=0$ sei

$$
N_{i}=-\delta_{i=}
$$

Das Problem soll durch Integraltransformationen gelöst werden. Zunächst werden die Gleichungen einer Laplace-Transformation im Zeitbereich unterworfen.

$$
u_{i}^{(1)}\left(\beta, x_{1}, x_{2}\right)=\int_{0}^{\infty} u_{i}\left(t, x_{1}, x_{2}\right) e^{-x^{i}} d z
$$

Das ergibt:

$$
\rho s^{2} u_{i}^{(2)}=\frac{\partial_{-u_{i}}^{(1)}}{\partial y_{j}}
$$

und

$$
\sigma_{i 2}^{(1)}=-N_{i(s)}^{(1)} \delta\left(x_{1}\right) \delta_{i 2}, \quad x_{2}=0 .
$$

Dann wird eine Raumtransformation

$$
u_{i}^{(2)}\left(s, k_{1}, k_{2}\right)=\int_{v} K\left(x_{1}, x_{2}, k_{1}, x_{2}\right) u_{i}^{(1)}\left(s_{1}, x_{1}, x_{2}\right) d x_{1} d x_{2}
$$

angewandt. Der Kern sei

$$
K=e^{k_{j} x_{j}}
$$


mit

$$
k_{1}=i k, k_{2}=-K
$$

Mit Anwendung des Satzes von Gauss ([7], [8]) ergibt sich:

$$
\begin{gathered}
u_{i}^{(2)}\left(s, k_{1}, k_{2}\right)=\frac{1}{\gamma_{1}}\left\{\left[N_{(s)}^{(1)}+p_{2} \int_{-\infty}^{+\infty} e^{i l x_{1}} u_{j}^{(1)} k_{j} d x_{1}\right] \delta_{i 2}+\right. \\
\left.+p_{1} k_{i} \int_{-\infty}^{+\infty} e^{i l x_{1}} u_{2}^{(1)} d x_{1}-p_{2} K \int_{-\infty}^{+\infty} e^{i k x_{1}} u_{i}^{(1)} d x_{1}\right\} \\
+\frac{p_{3} k_{i}}{\gamma_{1} \gamma_{2}}\left\{N_{(s)}^{(1)} k_{2}+p_{1} k_{j}^{2} \int_{-\infty}^{+\infty} e^{i k x_{1}} u_{2}^{(1)} d x_{1}-\right. \\
\left.-2 p_{2} K \int_{-\infty}^{+\infty} e^{i k x_{1}} u_{j}^{(1)} k_{j} d x_{1}\right\}
\end{gathered}
$$

Dabei sind zur Abkürzung:

$$
p_{1}(s)=\frac{(\gamma+s) \lambda+\beta}{\gamma+s} p_{2}(s)=\frac{(\gamma+s) \mu-\frac{j}{2} \beta}{\gamma+s} p_{3}(s)=p_{1}(s)+p_{2}(s)[15 a]
$$

und

$$
\gamma_{1}=\rho s^{2}-p_{2} k_{j}^{2}, \quad \gamma_{2}=\rho s^{2}-\left(p_{1}+2 p_{2}\right) k_{j}^{-}
$$

gesetzi worden.

Die Inversion in dem Originalbereich geschieht in 3 Schritten:
1.) $K \rightarrow x_{2}$
2.) $k \rightarrow x_{1}$
3.) $s \rightarrow t$.

Die erste Rücktranslormation, eine Laplace-Inversion, ist einfach.

Führt man

$$
\xi^{2}=k^{2}+\frac{\rho s^{2}}{p_{2}} . \quad n^{2}=k^{2}+\frac{\rho s^{2}}{p_{1}+2 p_{2}}
$$

ein, so erhält man: 


$$
\begin{aligned}
& \bar{u}_{1}{ }^{(1)}=-i \frac{p_{3}}{p_{2}\left(p_{1}+2 p_{2}\right)} N_{(-1)}^{(1)} \frac{\operatorname{Sin} \xi x_{2}}{\zeta} * \operatorname{Cos} \eta x_{2}+ \\
& +2 \frac{p_{j}}{p_{1}+2 p_{2}} k_{2} \int_{-\infty}^{+\infty} e^{i k x_{1}} u_{1}^{(1)} d x_{1} \frac{\operatorname{Sin} \xi x_{2}}{\xi} * \operatorname{Cos} \eta x_{2}+\int_{-\infty}^{+\infty} e^{i k x_{1}} u_{1}^{(1)} d x_{1} \operatorname{Cos} \xi x_{2}- \\
& -i K_{1} \int_{-\infty}^{+\infty} e^{i\left(x x_{1, \ldots}(1)\right.} d x_{1}\left(\frac{p_{1}}{p_{2}}-\frac{\operatorname{Sin} \xi x_{2}}{\xi}+\frac{p_{3}}{p_{1}+2 p_{2}} k^{2} \frac{\operatorname{Sin} \xi x_{2}}{\xi} * \frac{\left.\operatorname{Sin} \eta x_{2}\right)}{\eta}\right)+ \\
& +\frac{p_{3}}{p_{2}} \operatorname{Cos} \xi x_{2} * \operatorname{Cos} \eta x_{2} \\
& \bar{u}_{2}^{(1)}=-\frac{1}{p_{2}} N_{t \in 1}(1)\left(\frac{\operatorname{Sin} \xi x_{2}}{\xi}-\frac{p_{3}}{p_{1}+2 p_{2}} \operatorname{Cos} \xi x_{2} * \operatorname{Cos} \eta x_{2}\right)- \\
& -\imath k \int_{-\infty}^{+\infty} e^{i h x_{1}} u_{1}^{(1)} u_{1}^{(1)} d x_{1}\left(\frac{\operatorname{Sin} \xi x_{2}}{\xi}-2 \frac{p_{2}}{p_{1}+2 p_{2}} \operatorname{Cos} \xi x_{2} * \operatorname{Cos} \eta x_{2}+\right. \\
& +\int_{-\infty}^{+\infty} e^{i k x_{1}} u_{2}{ }^{(1)} d x_{1} \mid \operatorname{Cos} \xi x_{2}+\frac{p_{1} p_{3}}{p_{1}\left(p_{1}+2 p_{2}\right)} k^{2} \frac{\operatorname{Sin} \xi x_{2}}{\xi} * \operatorname{Cos} \eta x_{2}- \\
& \left.-\frac{p_{3}}{p_{2}} \eta \operatorname{Cos} \xi x_{2} * \operatorname{Sin} \eta x_{2}\right]
\end{aligned}
$$

Nach Ausrechnen der auftretenden Faltungen nehmen die Gleichungen die Form

$$
\bar{u}_{1}^{(1)}=\frac{1}{2}\left(T_{1}^{(1)} e^{\xi x_{2}}+T_{2}^{(1)} e^{-\xi x_{2}}+T_{2}^{(1)} e^{\eta x_{2}}+T_{4}^{(1)} e^{-\eta x_{2}}\right)
$$

und

$$
\bar{u}_{2}^{(1)}=\frac{1}{2}\left(T_{1}^{(2)} e^{\xi x_{2}}+T_{2}^{(2)} e^{-\xi x_{2}}+T_{3}^{(2)} e^{\eta x_{2}}+T_{4}^{(2)} e^{-\eta x_{2}}\right)
$$


an. Hierbei soll

$$
\operatorname{Re}(\xi)>0, \quad \operatorname{Re}(\eta)>0
$$

gelten. Dann muss

$$
\lim _{x_{1} \rightarrow \infty} u_{t}^{(1)}=0
$$

sein.

Das führt auf die Bedingungen

$$
T_{1}{ }^{(1)}=-T_{3}^{(1)}=0 \text { und } T_{1}^{(2)}=-T_{3}^{(2)}=0 .
$$

Aus diesen Bedingungen werden die Randwerte von $u_{i}^{(1)}$ bestimmt. Das Ergebnis ist schliesslich:

$$
\begin{gathered}
\bar{u}_{1}^{(1)}=-\frac{i}{?} \frac{k}{p_{2}} \frac{\xi \eta e^{-\xi x_{2}}+\zeta^{2} e^{-\eta x_{2}}}{\zeta^{-1}-h^{2} \zeta i j} N_{s}^{(1)} \\
\bar{u}_{2}^{(1)}=\frac{1}{2} \frac{\eta}{p_{2}} \frac{-k^{2} e^{-\xi x_{2}}+\zeta^{2} \rho^{-\eta x_{2}}}{\zeta^{-1}-k^{2} \xi \eta} N_{s}^{(1)}
\end{gathered}
$$

mit

$$
\zeta^{2}=k^{2}+\frac{1}{2} \frac{\zeta s^{2}}{p_{2}}
$$

Daraus folgt dann:

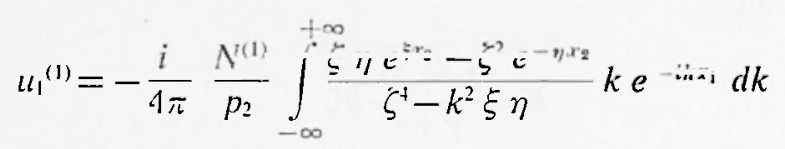

und

$$
u_{2}^{(1)}=-\frac{1}{4 \pi} \frac{N^{(1)}}{p_{2}} \int_{-\infty}^{+\infty} \frac{k^{2} e^{-\xi x_{2}}-\zeta^{2} e^{-\eta x_{2}}}{\zeta^{4}-k^{2} \xi \eta} \eta e^{-i k x_{1}} d k
$$

Die Integranden besitzen die Verzweigungspunkte $\xi=0$ und $\eta=0$, sowie einen Pol $\zeta^{4}-k^{2} \xi \eta=0$. Sie liegen in der komplexen $k$-Ebene auf der imaginären Achse. 
Zur Durchführung der Integration werden die Integranden in jeweils zwei Summanden aufgeteilt, die je besonders behandelt werden.

$$
\begin{aligned}
& F_{11}^{(1)}=-\frac{i}{4 \pi} \frac{N^{(1)}}{p_{2}} \int_{-\infty}^{+\infty} \frac{\xi \eta k}{R} e^{\left(-i k x_{1}-\xi x_{2}\right.} d k \\
& F_{21}{ }^{(1)}=-\frac{1}{4 \pi} \frac{N^{(1)}}{p_{2}} \int_{-\infty}^{+\infty} \frac{k^{2} \eta}{R} e^{\left.\prime-i k x_{1}-\xi x_{2}\right)} d k \\
& F_{i 2}{ }^{(1)}=+\frac{i}{4 \pi} \frac{N^{(1)}}{p_{2}} \int_{-\infty}^{+\infty} \frac{\zeta^{2} k}{R} e^{\left(-i k x_{1}-\eta x_{2}\right)} d k \\
& F_{22}{ }^{(1)}=+\frac{1}{4 \pi} \frac{N^{(1)}}{p_{2}} \int_{-\infty}^{+\infty} \frac{\zeta^{2} \eta}{R} e^{\left(-i k_{1}-\eta x_{2}\right)} d k
\end{aligned}
$$

Dabei gilt:

$$
R=\zeta^{+}-k^{2} \xi \eta
$$

Zur Behandlung von $F_{12}{ }^{(1)}$ und $F_{22}{ }^{(1)}$ wird die Transformation

$$
k=-i \frac{s}{a} \operatorname{Cos} \varphi
$$

durchgeführt, mit

$$
\bar{k}^{2}=\frac{2 \gamma+3 s}{\gamma+s} b^{2},
$$

und der willkürlichen Annahmen

$$
\frac{a^{2}}{b^{2}}=3, \frac{\xi}{\delta}=\frac{\tilde{i}}{2} b^{2} \quad \text { (s. }[4 \mid)
$$

und es werden die kartesischen Koordinaten $x_{1}, x_{2}$ durch Polarkoordinaten $r, \varphi$ ersetzt:

$$
x_{1}=r \cos \varphi, \quad x_{2}=r \sin \varphi .
$$


Die Integrale konvergieren, wenn der Integrationsweg in dem Teil der komplexen $\psi$-Ebene verläuft. Er wird durch

$$
0 \leq \psi_{i} \leq \frac{\pi}{2} \text { für } 0 \leq \psi<\frac{\pi}{2} \text { und } \frac{\pi}{2} \leq \psi_{i} \leq \pi \text { für } \frac{\pi}{2}<\varphi \leq \pi
$$

bestimmt. Der Pol liegt auf dem Verzweigungsschnitt.

Durch Anwendung des Satzes von Cauchy wird es möglich, den Integrationsweg durch den Sattelpunkt zu legen. Dieser liegt in

$$
\psi_{r}=\operatorname{Re}(\psi)=0, \quad \psi_{1}=\operatorname{Im}(\psi)=\varphi
$$

Nimmt man $r \times A$ an, so führt nun die Sattelpunktsmethode zu den Lösungen:

$$
F_{i 2}{ }^{(1)}=\frac{i}{2}-\frac{N^{(1)}}{p_{2}} \sqrt{\frac{s}{2 \pi a r}} \sin \varphi \frac{\zeta_{s}^{2} k_{s}}{\zeta_{s}^{4} k_{s}^{2} \xi_{s}}-e^{-8 \frac{v}{a}}
$$

und

$$
F_{22}^{(1)}=\frac{1}{i} \frac{N^{(1)}}{p_{2}} \sqrt{\frac{s}{2 \pi \bar{a} r}} \sin \varphi \frac{\zeta_{s}^{2} \eta_{.}}{\Gamma_{s}} e^{-\frac{v}{\bar{a}}}
$$

Dabei sind die mit dem Index $s$ bezeichneten Grössen die Werte dieser Grössen im Sattelpunkte.

Zur Berechnung von $F_{11}{ }^{(1)}$ und $F_{01}^{(1)}$ dient die Transformation

$$
k=-i \frac{s}{b} \operatorname{Cos} X
$$

mit

$$
\bar{b}^{2}=\frac{\gamma+4 s}{\gamma+s} b^{2}
$$

Der Sattelpunkt wird dabei

$$
X_{r}=0, \quad X_{i}=\varphi
$$

gegeben. In diesem Falle kann der Sattelpunkt auf dem Verzweigungsschnitt liegen. Das ist der Fall, falls 


$$
0 \leq \varphi \leq a n \cos \frac{\bar{b}}{\bar{a}} \text { oder } \pi-\text { an } \cos \frac{\bar{b}}{\bar{a}} \leq \varphi \leq \pi
$$

gilt. Dann wird als Integrationsweg durch den Sattelpunkt der Weg

$$
-\cos <X_{r} \leq 0, X_{i}=\varphi \text { und } 0 \leq X_{r} \cos , X_{i}=\varphi
$$

gcwählt. Der gleiche Weg führt auch zum Ziele, wenn der Sattelpunkt nicht auf dem Verzweigungsschnitt liegt.

Das Ergebnis ist in beiden Fällen

$$
\begin{aligned}
& F_{11}^{(1)}=-\frac{i}{4} \sqrt{\frac{s}{2 \pi \bar{b} r}} \frac{N^{(1)}}{p_{2}} \sin \varphi\left[\left(\frac{\xi_{s} \eta_{s} k_{s}}{R_{s}} e^{-8 \frac{v}{\bar{l}}}+\left(\frac{\xi_{s} \eta_{s} k_{s}}{R_{s}}\right) e^{-s \frac{v}{\bar{l}}}\right]\right. \\
& F_{21}^{(1)}=-\frac{1}{4} \sqrt{\frac{s}{2 \pi \bar{b} r}} \frac{N^{(1)}}{p_{2}} \sin \varphi\left[\left(\frac{\eta_{s} k_{s}^{2}}{R_{s}}\right)_{l} e^{-s \frac{v}{\imath}}+\left(\frac{\eta_{s} k_{s}^{2}}{R_{s}}\right) e_{v}^{-* \frac{v}{l}}\right]
\end{aligned}
$$

Dabei bedeuten die Indizes $l$ und $r$, dass im ersten Falle die Sattelpunktswerte am linken Ufer $\left(X_{,}<0\right)$, bzw. am rechten Ufer $\left(X_{r}>0\right)$ des Verzweigungsschnittes zu wählen sind. Diese Werte stimmen natürlich iiberein, wenn der Sattelpunkt nicht auf dem Verzwcigungsschnitt liegt.

Falls der Sattelpunkt auf dem Verzweigungsschnitt liegt, d. h. für den Fall der Ungleichungen [35] ist die Lösung noch durch zwei Integrale längs des linken und des rechten Ufers des Verzweigungsschnittes vom Sattelpunkte bis zum Verzweigungspunkt $\cos X_{i}= \pm l / a$ zu ergänzen. Diese Integrale werden mit $F_{11}^{(1)}$ und $F_{21}{ }^{(1)}$ bezeichnet und später behandelt werden.

Um nun die Inversion im Zeitbereich durchzuführen, muss zunächst $N_{(s)}{ }^{(1)}$ berechnet werden.

Aus

$$
N(t)=\operatorname{Im}\left(e^{i \omega t}\right)
$$

ergibt sich:

$$
N_{(s)}{ }^{(1)}=\frac{\omega}{s^{2}+\omega}
$$


Die Inversion ist dann:

$$
F_{i j}=\frac{\omega}{2 \pi i} \int_{s_{2}-i \infty}^{s_{0}-i \infty} F_{i j}^{(1)} e^{s t} \frac{d s}{s^{2}+\omega}
$$

Dabei nehmen $F_{12}$ und $F_{22}$ die Form

$$
F=\frac{1}{2 \pi i} \int_{s_{0}-i \infty}^{s_{2}+\infty} A(s) e^{s(t-r / a)} d s
$$

an.

Sie stellen damit die Kompressionswelle

$$
u_{i}{ }^{k}=F_{i 2}
$$

dar.

Entsprechend erhalten $F_{11}$ und $F_{21}$ die Form

$$
F=\frac{2 \pi i}{1} \int_{s_{1}-i \infty}^{s_{1}-i \infty} A_{(s)} e^{-s(t-r \bar{l})} d s .
$$

Sie stellen also die Scherwelle

$$
u_{i}^{s}=F_{i 1}
$$

dar. Die Integration längs des Verzweigungsschnittes liefert die geführte Welle, die aber erst in einer späteren Arbeit behandelt werden soll.

Zur Auswertung der Fij muss man die Verzweigungspunkte, die Pole und die Sattelpunkte in der komplexen s-Ebene bestimmen. Das bereitet keine grundsätzlichen Schwierigkeiten. Das Sattelpunktsverfahren ist wieder anwendbar. Eine Abschätzung der Integrale durch die Sattelpunkte scheint zu ergeben, dass sie keine grosse Rolle spielen. Dasselbe gilt für die Pole $\rho=0$. Die endgülttige Berechnung jener Lösungsanteile soll einer späteren, ausführlicheren Arbeit vorbehalten werden. Hier sollen lediglich diejenigen Anteile der Lösung behandelt werden, welche sich aus den Polen $s= \pm i \omega$ ergeben. Sie sind auch deshalb von besonderem Interesse, weil die ihnen entsprechenden Wellen gerade das eingeprägte Signal transportieren. 
Die Kompressionswelle und die Scherwelle sollen vergleichend betrachtet werden. Bei der Behandlung dieser Wellen erhält man Lüsungsformen der Art:

$$
F=A(\omega) e^{i \omega(1-\nu / \nu(\omega))}-\delta \nu
$$

Der Hauptaugenmerk soll auf die Berechnung des Exponenten der $e$ Funktion gelegt werden, da dieser die interessanten Informationen über Geschwindigkeit und Extinktion der Wellen enthält. Die Berechnung selbst ist eine reine Routinearbeit, die nicht dargelegt werden muss. Die Ergebnisse für die Kompressions-und die Scherwelle werden in den Figuren 1 und 2 vorgelegt. Dabei ist die dimensionslose Geschwindigkeit

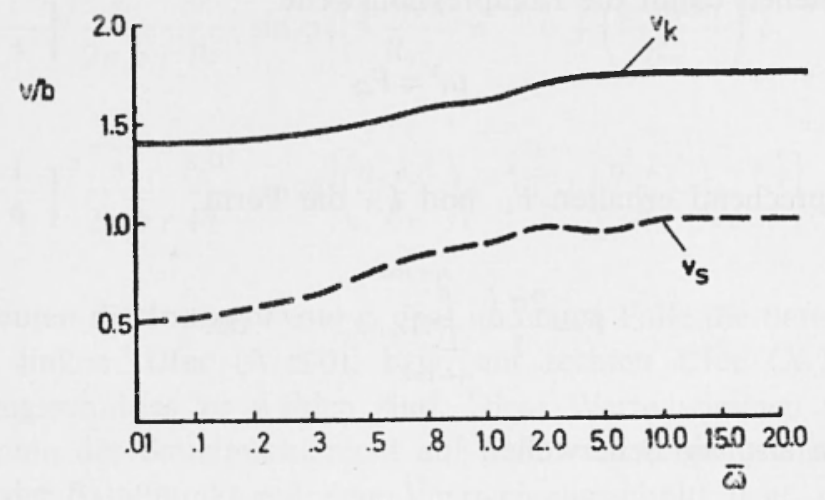

Fig. 1

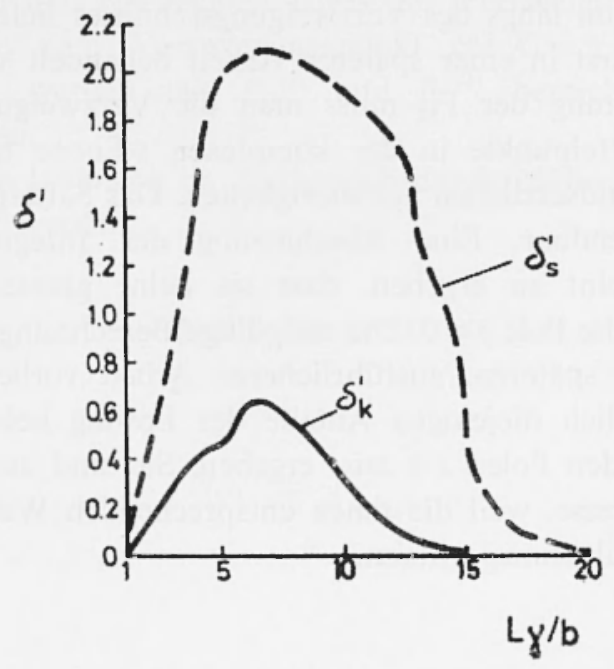

Fig. 2 
$\vartheta / b$ als Funktion der dimensionslosen Frequenz $a / \gamma=\omega$ dargestellt worden. Für sehr grosse Frequenzen nähert sich die Geschwindigkeit der Kompressionswelle jener der Kompressionswellen in ideal elastische Materialien bei adiabatischen Vorgängen. Sind die Frequenzen sehr klein, so stellt sich ein Grenzwert der Geschwindigkeit ein, der bei isothermen Vorgängen zu beobachten wäre. Dieses Verhalten war zu erwarten. Die entsprechende Feststellung gilt für die Scherwelle.

Auch rür die Extinktion wird eine dimensionslose Darstellung gewählt. Statt der Wellenlänge $L$ wird die dimensionslose Grösse $L \gamma / b$ benutzt und die Extinktion wird auf die Wellenlänge bezogen. Damit ist die in Fig. 2 auftretende Grösse $\delta$ eine dimensionslose Extinktionskonstante. Für sehr grosse und für sehr kleine Wellenlängen wird die Extinktion sehr klein, was zu erwarten ist. Das Maximum der Extinktion pro Wellenlänge ist bei der Scherwelle beträchtlich grösser als bei der Kcmpressionswelle. Auch diese Feststellung ist einleuchtend. Es muss an dieser Stelle daraul hingewiesen werden, dass sowohl das Verhalten der Geschwindigkeiten, wie auch das der Extinktion von der Form des Signals abhängig isı (s. [41).

Die Poissonkonstante ist nur für die Grenzfälle sehr grosser und sehr kleiner Frequenzen berechnet worden. Im ersten Falle erhält man natürlich den Wert 0,25. Im zweiten Falle ergibt sich $\sigma=0,429$.

Es soll nun die Schwingungsrichtung der Wellen betrachtet werden. Peide Komponenten der Kompressionswelle sind proportional zu $\sin \varphi$. Da nun $k_{s}$, wie leicht $\mathrm{zu}$ berechnen ist, proportional $\mathrm{zu} \cos \varphi$ und $\eta_{s}$ proportional zu $\sin \varphi$ ist, so folgt aus [31a] und [31b], dass die Kompressicnswelle in grosser Entfernung vom Ursprung rein longitudinal ist. Am stärksten ist die radiale Komponente in vertikaler Richtung. In der Oberfläche verschwindet sie. Die Komponenten der Scherwelle sind ebenfalls proportional zu $\sin \varphi$. Auch hierbei ist wieder $k_{s} \mathrm{zu} \cos \varphi$ proportional, $\ddot{\xi}_{s} z u \sin \varphi$.

Dann folgt aus [37a] und $|37 \mathrm{~b}|$, dass die Scherwelle eine reine $S V$-Welle ist, deren Tangentialkomponente am stärksten in der Oberfläche ist und in vertikaler Richtung verschwindet.

Alle mitgeteilten Ergebnisse sind anschaulich evident. Eine numerische Berechnung des Amplitudenverlaufes ist geplant. Es ist auch beabsichtigt, andere Bezcichungen zwischen $\beta$ und $\gamma$ der Rechnung zu Grunde zu legen. 


\section{LITERATUR}

Fri:udenthal, A. M. u. Geiringer, H., 1958. - The mathematical theories of the inelastic Continuum, in Handbuch der Physik Bd. VI, Herausgegeben von Flügge. S., S. 257.

Deriaguine, B., 1954. - Propagation des ondes élastiques dans les milieux non idéalement élastiques. Beiträge z. angew. Geoph. 4, S. 457.

Sokoloff, P. T. u. Skriabin, V. J., 1937. Experimental and theoretical investigations on dispersion and absorption of elastic waves. Gerlands Beiträge $z$. Geoph. 49, S. 165.

Menzel, H., 1954. - On the propagation of seismic waves in a solid body with elastic afterworking. Geophysical prospecting, Bd. IV, S. 139.

MENZEL, H., 1954. - On the stress-strain relation in a not perfectly elastic solid body. Extrait des publications du Bureau Central Seismologique International, Série A, Travaux Scientifiques, Fascicule 19, S. 125.

SzElw1S, R., 1973. - Ein Verfahren zur Lösung des ersten Randwertproblems der Elastodynamik angewandt auf den Zylinder. Hamburger Geophysikalische Einzelschriften, Heft 22.

R.IIAL, M., 1977. - Wellen im Keil als Randwerlaufgabe der Elastodynamik. Dissertation in der Universität Hamburg. 